\title{
Digitale Innovationen und Technologiesouveränität
}

\author{
Digitale Innovationen verändern zunehmend Wirtschaft und Gesellschaft. Es ist Aufgabe der \\ Politik, diese Veränderungen im Dialog mit der Gesellschaft und im Einklang mit unseren \\ Werten zu gestalten. Technologiesouveränität ist dafür eine wichtige Voraussetzung. \\ Dabei geht es nicht um die Abschottung von internationalen Märkten, sondern um \\ die Fähigkeit, Anforderungen an Technologien zu formulieren und diese am Markt \\ durchzusetzen. Einer integrierten Forschungs- und Innovationspolitik, die die Förderung von \\ Technologieentwicklung, Technologietransfer und Zukunftskompetenzen gleichermaßen in den \\ Blick nimmt und auf europäische Zusammenarbeit setzt, kommt dabei eine zentrale Rolle zu.
}

\begin{abstract}
Die digitale Transformation von Wirtschaft und Gesellschaft bestimmt derzeit in hohem Maße die politische und gesellschaftliche Debatte. Dabei sind die zugrundeliegenden Innovationen und Technologien nicht neu. Vor gut 50 Jahren - am 29. Oktober 1969 - lief die erste elektronische Nachricht über das ARPANET (Advanced Research Projects Agency Network), den technischen Vorläufer des Internets. Und bereits 1941 entwickelte Konrad Zuse mit dem Z3 den ersten funktionsfähigen digitalen Computer und damit Grundprinzipien, die sich auch in heutigen PCs wiederfinden. Selbst das Forschungsgebiet der Künstlichen Intelligenz (KI) geht auf eine Konferenz am Dartmouth College von 1956 zurück. Und auch die Forschungspolitik hat das Thema frühzeitig erkannt und vorangetrieben: Förderprogramme und -strategien des Bundes für die Erforschung, Entwicklung und Anwendung von Informations- und Kommunikationstechnologien lassen sich (mindestens) bis in die 1980er Jahre zurückverfolgen.

Was also treibt die aktuelle Debatte? Drei Entwicklungen sind hier maßgeblich: Erstens haben sich die zugrundeliegenden Schlüsseltechnologien (vgl. Abbildung 1) in den zurückliegenden Jahren rasant weiterentwickelt. Dies betrifft die eingesetzte Hardware, Software und Kommuni-
\end{abstract}

Prof. Dr.-Ing. Ina Schieferdecker ist Leiterin der Abteilung Forschung für Digitalisierung und Innovationen im Bundesministerium für Bildung und Forschung (BMBF) in Berlin.

Dr. Christoph March ist dort Referent für Grundsatzfragen, Digitalisierung und Transfer. kationstechnologien, aber auch deren Zusammenspiel in komplexer werdenden Systemen. So hat das Moore'sche Gesetz - die 1965 von Gordon E. Moore vorausgesagte Verdopplung der Zahl an Transistoren auf einem Chip alle 18 Monate - bis heute Bestand. ${ }^{1}$ Eingebettete Systeme, als Basis für die Vernetzung von Maschinen und Objekten im „Internet der Dinge“ (IoT), wurden so erst ermöglicht. Folge der zunehmenden Vernetzung ist eine drastisch steigende Datenmenge: Gemäß aktueller Schätzungen wurden allein im Jahr 2018 weltweit Daten im Umfang von 33 Zettabyte (33 Billionen Gigabyte) erzeugt, ein zunehmender Teil davon in Echtzeit. ${ }^{2}$ Neue Kommunikationstechnologien wie Glasfasernetze oder $5 \mathrm{G}$ sind für deren Übertragung essenziell. Gleichzeitig wurden auch die Methoden und Werkzeuge zur Datenanalyse, wie Methoden der Künstlichen Intelligenz und zu Big Data, kontinuierlich weiterentwickelt. Sie ermöglichen in Kombination mit verbesserter Sensorik und Aktorik eine zunehmende Kognitionsfähigkeit und Autonomie technischer Systeme. Die steigende Vernetzung und Virtualisierung der physischen Welt, wachsende Fähigkeiten technischer Systeme zur Kognition und Autonomie sowie der dadurch getriebene Wissenszuwachs in allen Wissenschafts- und Forschungsbereichen sind Kerncharakteristika des digitalen 
Abbildung 1

Schlüsseltechnologien der Digitalisierung
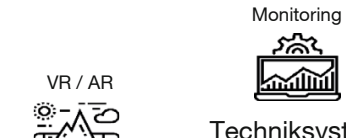

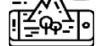

Blockchain
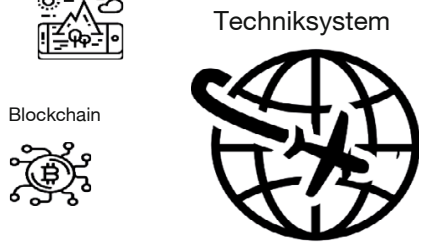

IoT
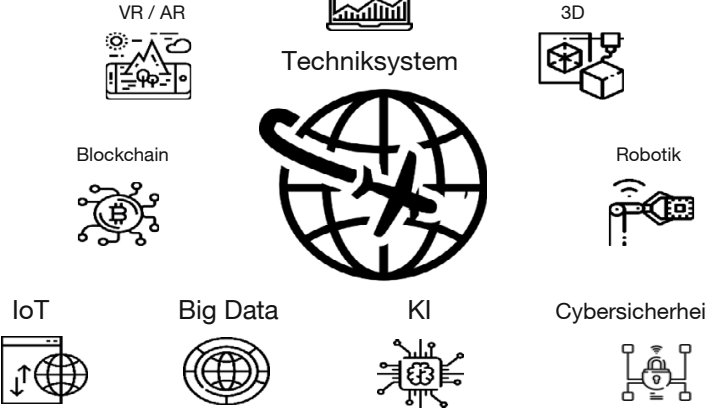

Cybersicherheit

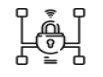

Quellen: Wissenschaftlicher Beirat der Bundesregierung Globale Umweltveränderungen (WBGU): Unsere gemeinsame digitale Zukunft, Hauptgutachten, Berlin 2019, S. 70; Symbole von https://www.flaticon. com/de/ (28.2.2020).

Zeitalters und bestimmen maßgeblich die Interaktion technischer Systeme mit zivilisatorischen Lebensbereichen. ${ }^{3}$

Zweitens sind die frühen digitalen Innovationen, wie der PC, das Internet oder das Mobiltelefon, in die Breite von Wirtschaft und Gesellschaft diffundiert. In Deutschland beträgt der Ausstattungsgrad der privaten Haushalte mit diesen Technologien mittlerweile über $90 \% .{ }^{4}$ In allen Ländern der Organisation für wirtschaftliche Zusammenarbeit und Entwicklung (OECD) sind ähnliche Durchdringungsraten zu verzeichnen. ${ }^{5}$ Gleichzeitig hat die Diffusion fortgeschrittener Technologien bereits begonnen. Mit 338 Robotern je 10000 Beschäftigten in der Industrie lag Deutschland im Jahr 2018 weltweit auf Rang 3, knapp vor Japan (327 Roboter je 10000 Beschäftigten) und den USA (217) aber noch deutlich hinter Singapur (831) und Südkorea (774). ${ }^{6}$

Drittens werden die Auswirkungen der Entwicklung und Verbreitung digitaler Technologien auf Wirtschaft und Gesellschaft mittlerweile deutlich spürbar. Am klarsten zeigt sich dies am Markterfolg der amerikanischen Big-Five-Unternehmen Google/Alphabet, Apple, Facebook, Amazon, Microsoft und zunehmend auch chinesischer Unternehmen

3 Vgl. Wissenschaftlicher Beirat der Bundesregierung Globale Umweltveränderungen (WBGU): Unsere gemeinsame digitale Zukunft, Hauptgutachten, Berlin 2019, S. 92 ff.

4 Vgl. Bundesamt für Statistik: Ausstattung privater Haushalte mit Informations- und Kommunikationstechnik, 2019, https://www.destatis. de/DE/Themen/Gesellschaft-Umwelt/Einkommen-Konsum-Lebensbedingungen/Ausstattung-Gebrauchsgueter/Tabellen/liste-infotechnik-d.html (10.12.2019).

5 Vgl. WBGU, a.a. O., S. $53 \mathrm{ff}$.

6 Vgl. International Federation of Robotics (IFR): World Robotics 2019 - Industrial Robots, https://ifr.org/downloads/press2018/IFR\%20 World\%20Robotics\%20Presentation\%20-\%2018\%20Sept\%202019. pdf (10.12.2019). wie Tencent und Alibaba. Mit Hilfe digitaler Technologien und neuer Geschäftsmodelle haben diese innerhalb kürzester Zeit ein drastisches Wachstum von Kapital und Umsatz erreicht und zählen heute zu den Unternehmen mit der höchsten Marktkapitalisierung weltweit.? Parallel ändern digitale Lösungen die Arbeitsmärkte, ${ }^{8}$ das Konsumverhalten ${ }^{9}$ und die Verbreitung von Informationen, etwa in Form von „Fake News" ${ }^{\text {"10, }}$, und bieten neue Interaktionsformen, beispielsweise auf sozialen Plattformen.11

\section{Gestaltungsanspruch der Politik}

Es ist Aufgabe der Politik, diesen Entwicklungen mit spürbarem Gestaltungsanspruch im Dialog mit der Gesellschaft und im Einklang mit deutschen (und europäischen) Werten eine Richtung zu geben. Im Vordergrund steht dabei die Verbesserung der Lebensbedingungen der Bürgerinnen und Bürger, nicht die Reaktion auf einen Trend oder singuläre wirtschaftliche Interessen. Deutschland (und Europa) muss dabei einen eigenen Weg finden. Wir können weder den chinesischen Weg des staatlichen Totalitarismus gehen noch Unternehmen die Gestaltungsmacht überlassen, wie in den USA. Hierfür gibt es zwei wesentliche Gründe:

1. Zum einen folgt bei uns das Recht auf informationelle Selbstbestimmung aus dem im Grundgesetz (GG) verankerten allgemeinen Persönlichkeitsrecht (Art. 2 Abs. 1 GG in Verbindung mit Art. 1 Abs. 1 GG). ${ }^{12}$ Es beinhaltet insbesondere die Entscheidungsfreiheit jedes Einzelnen über die Nutzung und Weitergabe personenbezogener oder personenbeziehbarer Daten. Dieses Recht wird im digitalen Zeitalter herausgefordert, da insbesondere Unternehmen einen Anspruch auf personenbezogene und -beziehbare Daten als Grundlage ihrer Geschäftsmodelle erheben. Der Staat hat hier eine Schutzfunktion gegenüber seinen Bürgerinnen und Bürgern. Ziel der Politik muss es sein, individuelle Freiheitsrechte, wie das Recht auf informationelle Selbstbestimmung, aber auch auf Meinungsfreiheit und Schutz vor Diskriminierung und Manipulation, im digitalen Zeitalter weiterhin zu gewähr-

7 Vgl. PricewaterhouseCoopers: Global Top 100 Companies by Market Capitalisation, 2019, https://www.pwc.com/gx/en/audit-services/publications/assets/global-top-100-companies-2019.pdf (10.12.2019).

8 Vgl. D. Acemoglu, P. Restrepo: Robots and jobs: Evidence from US labor markets, NBER working paper, Nr. w23285, 2017.

9 Vgl. J. Waldfogel: How Digitization Has Created a Golden Age of Music, Movies, Books, and Television, in: Journal of Economic Perspectives, 31. Jg. (2017), H. 3, S. 195-214.

10 Vgl. H. Allcott, M. Gentzkow: Social Media and Fake News in the 2016 Election, in: Journal of Economic Perspectives, 31. Jg. (2017), H. 2, S. 211-236.

11 Vgl. M. Luca: User-Generated Content and Social Media, in: S. P. Anderson, J. Waldfogel, D. Stromberg (Hrsg.): Handbook of Media Economics, Bd. 1A, 2016, S. 205-224.

12 Vgl. Neue Juristische Wochenschrift: Verfassungsrechtliche Überprüfung des Volkszählungsgesetzes 1983, 1984, H. 8, S. 419-428. 
leisten. Darüber hinaus hat der Staat auch eine Schutzfunktion für die Marktwirtschaft, die durch eine zunehmende Kapitalisierung von Daten und ihre Monopolisierung herausgefordert ist.

2. Zum anderen unterscheidet sich unser Wirtschafts- und Sozialstaatmodell substanziell vom US-amerikanischen und chinesischen Ansatz. Inklusives Wachstum ist in Deutschland und Europa ein zentrales politisches Ziel. Dies spiegelt sich, im Vergleich zu China und den USA, beispielsweise in einer höheren Staatsquote und in der Folge in niedrigeren Maßen für Ungleichheit nach erfolgter Umverteilung wider (vgl. Abbildung 2). Aktuelle Studien legen dabei einen Zusammenhang zwischen dem Grad an Umverteilung und der Fähigkeit, besonders risikoreiche, disruptive Innovationen hervorzubringen, nahe. ${ }^{13}$ In der Tat liegt ein solcher Zusammenhang auch empirisch nah, wenn beispielsweise Zahlen zu Gründungsraten oder der Verfügbarkeit von Wagniskapital herangezogen werden. Abbildung 2 stellt dies exemplarisch anhand des Gini-Koeffizienten, einem statistischen Maß zur Darstellung von Ungleichverteilungen, berechnet für die Einkommen nach Steuern und Transferleistungen (gemäß Daten der „Standardized World Income Inequality Database“, SWIID ${ }^{14}$ ) und des jährlich verfügbaren Wagniskapitals als Anteil am Bruttoinlandsprodukt (gemäß Daten der OECD ${ }^{15}$ ) dar.

So begründet sich ein Politikansatz, der die eigenen Stärken und Werte in den Vordergrund stellt und sich nicht in einen „Disruptionswettlauf“ mit den USA oder China begibt. Die Voraussetzungen, sich mit einem solchen Ansatz im globalen Wettbewerb zu behaupten, sind dabei alles andere als schlecht. Das Weltwirtschaftsforum bescheinigt Deutschland in seinem Globalen Wettbewerbsbericht 2019 im zweiten Jahr in Folge den ersten Platz bei der Innovationsfähigkeit. ${ }^{16}$ Und zudem kommen sechs der zehn besten Nationen aus Europa.

\section{Technologiesouveränität im digitalen Zeitalter}

Eine wesentliche Voraussetzung, um den Gestaltungsanspruch der Politik praktisch umzusetzen, ist die sogenannte Technologiesouveränität. Dabei geht es nicht um eine Ab-

13 Vgl. z.B. D. Acemoglu, J. A. Robinson, T. Verdier: Asymmetric Growth and Institutions in an Interdependent World, in: Journal of Political Economy, 125. Jg. (2017), H. 5, S. 1245-1303.

14 Vgl. F. Solt: The Standardized World Income Inequality Database, Version 8, 2019, https://doi.org/10.7910/DVN/LM4OWF (28.2.2020), Harvard Dataverse, V3.

15 Für China vgl. A. Lu, J. Chen, F. Fu: China's Venture Capital (VC): Bigger than Silicon Valley's?, INSEAD 2018, https://www.insead.edu/ sites/default/files/assets/dept/centres/gpei/docs/insead-studentchina-venture-capital-apr-2018.pdf (30.12.2019).

16 Vgl. K. Schwab: The Global Competitiveness Report 2019, World Economic Forum, 2019, http://www3.weforum.org/docs/WEF_TheGlobalCompetitivenessReport2019.pdf (12.12.2019).
Abbildung 2

Ungleichheit und Wagniskapital im Ländervergleich

Wagniskapital als Anteil in \% am BIP

(2018 oder letztes verfügbares Jahr)

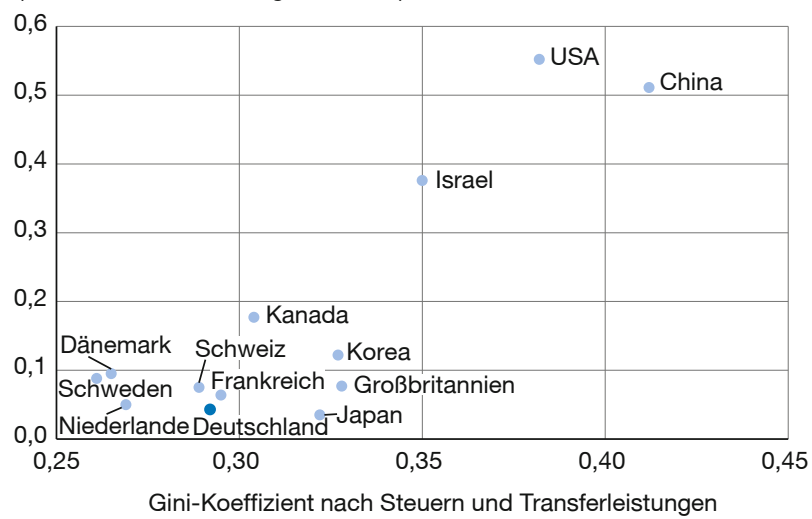

Gini-Koeffizient nach Steuern und Transferleistungen (2017 oder letztes verfügbares Jahr)

Quelle: eigene Darstellung nach Zahlen der SWIID und der OECD.

schottung von internationalen Märkten oder den Anspruch, technologische Autarkie zu erreichen: Die Vorteile des internationalen Handels, in Form von Wohlfahrtsgewinnen für alle beteiligten Länder, haben sich in der Realität im Großen und Ganzen bestätigt. ${ }^{17}$ Sie sind gerade für Deutschland als Exportnation evident.

Daher definiert ein anderer Ansatz Souveränität als Fähigkeit eines Staats, die Selbstbestimmung seiner Bürgerinnen und Bürger zu gewährleisten. ${ }^{18}$ Technologiesouveränität ist dabei eine Grundlage, um das Recht auf informationelle Selbstbestimmung effektiv zu schützen und durchzusetzen. Konkret geht es darum, auf Basis der gesetzten Gestaltungsziele Anforderungen an Technologien und Systemlösungen zu formulieren - etwa hinsichtlich IT-Sicherheit, Datenschutz, Zuverlässigkeit, Interoperabilität oder Nachhaltigkeit - und diese am Markt auch durchzusetzen. Letzteres umfasst beispielsweise, die Entwicklung europäischer Standards zu unterstützen und weltweit voranzutreiben sowie Kompetenzen zu entwickeln, um importierte Komponenten auf die Erfüllung der Anforderungen hin zu überprüfen und in komplexe Systeme zu integrieren. Technologiesouveränität steht damit nicht im Konflikt mit dem Freihandel, sondern ermöglicht inn und steht Informationsasymmetrien entgegen, die Wohlfahrtsgewinne mindern oder gar verhindern können. ${ }^{19}$

17 Vgl. J. Südekum: Globalisierung unter Beschuss. Eine Bestandsaufnahme des Freihandels, in: Aus Politik und Zeitgeschichte, 68. Jg. (2018), H. 4-5, S. 4-10.

18 Vgl. R. Voigt: Staatliche Souveränität. Zu einem Schlüsselbegriff der Staatsdiskussion, Wiesbaden 2016, S. 5 f.

19 Vgl. D. Martimort, T. Verdier: Optimal domestic regulation under asymmetric information and international trade: a simple general equilibrium approach, in: The RAND Journal of Economics, 43. Jg. (2012), H. 4, S. 650-676. 
Abbildung 3

Eckpunkte der Technologiesouveränität

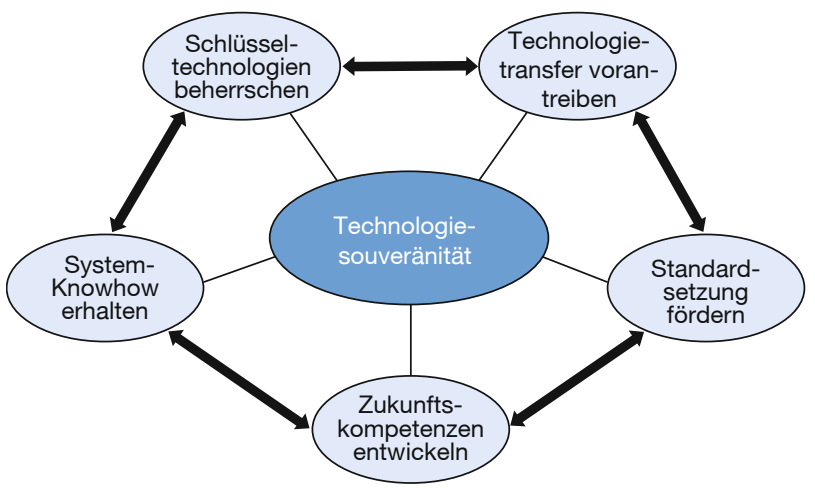

Quelle: eigene Darstellung.

Aus der Definition von Technologiesouveränität folgen zentrale Elemente einer staatlichen Technologie- und Innovationspolitik (vgl. Abbildung 3), um den Souveränitätsanspruch umzusetzen. Dabei geht es erstens um eine Beherrschung der relevanten Schlüsseltechnologien. Dies umfasst sowohl ein tiefgehendes Verständnis der Technologien als auch die Fähigkeit, diese weiterzuentwickeln, in komplexe Systeme zu integrieren und zur zweckgebundenen Anwendung zu führen. Zweitens geht es um den erfolgreichen Transfer der Technologien und ihrer Anwendungen in die breite Nutzung. Dadurch werden nicht nur Wertschöpfungspotenziale umfassend realisiert. Technologietransfer ist auch die Voraussetzung für eine Manifestierung von Standards, die sich häufig erst im Marktwettbewerb durchsetzen. Und drittens ist auch die Entwicklung der notwendigen Kompetenzen eine zentrale Voraussetzung für Technologiesouveränität. So setzt ein erfolgreicher Technologietransfer adäquat ausgebildete Fachkräfte voraus, die mit den neuen Technologien umgehen und sie an die Erfordernisse von Anwendungsfeldern in der Wirtschaft, Wissenschaft oder Zivilgesellschaft anpassen können. So sind auch Forscherinnen und Forscher gefordert, die den durch die neuen Methoden ermöglichten Wissenszuwachs über Forschungsfelder hinweg erst realisieren. Und schließlich sind Kompetenzen im Umgang mit digitalen Technologien eine wesentliche Voraussetzung für alle Bürgerinnen und Bürger, um nicht nur das Recht auf informationelle Selbstbestimmung wahrnehmen zu können, sondern ebenso die Vorteile der digitalen Technologien für das eigene Tun zu nutzen und kompetent mit den verbundenen Risiken umzugehen.

\section{Beispiele für eine gestaltende Innovationspolitik}

Mit Industrie 4.0 hat die deutsche Innovationspolitik gezeigt, dass sie dem Anspruch, das digitale Zeitalter zu gestalten und Technologiesouveränität zu gewährleisten, gerecht werden kann. Deutschland hat hier einen eigenen Trend etabliert, der auch international anerkannt wird. ${ }^{20}$ Dies zeigt sich sowohl in der weltweiten Verbreitung des Ansatzes als auch an den internationalen Investitionen in deutsche Industrie-4.0-Unternehmen. ${ }^{21}$

Der Erfolg beruht auf verschiedenen Säulen: Industrie 4.0 baut auf den Stärken Deutschlands im Maschinen- und Anlagenbau, in der Elektronikindustrie sowie bei Soft- und Hardware für eingebettete Systeme auf. Zudem wurde frühzeitig eine Zusammenarbeit zahlreicher Stakeholder aus Wirtschaft, Wissenschaft, Politik, Gewerkschaften und Verbänden etabliert. Die 2013 gestartete Plattform Industrie 4.0 umfasst heute mehr als 350 Stakeholder, die gemeinsam Lösungen in den Bereichen Referenzarchitekturen, Standardisierung und Interoperabilität, Technologie- und Anwendungsszenarien, Sicherheit vernetzter Systeme, rechtliche Rahmenbedingungen, Arbeit, Ausund Weiterbildung sowie digitale Geschäftsmodelle erarbeiten. ${ }^{22}$

Die Bundesregierung hat darüber hinaus die Entwicklung und Umsetzung von Industrie 4.0 mit inrer Forschungsund Innovationspolitik konsequent vorangetrieben. Gefördert wurden dabei die zugrundeliegenden Technologien (unter anderem Mikroelektronik, Softwaresysteme, Produktionstechnologien), der Transfer dieser Technologien in die Anwendung durch gezielte Maßnahmen zur Vernetzung (z. B. im Spitzencluster „It's OWL") und zur Unterstützung kleiner und mittlerer Unternehmen (z. B. Testbeds für Industrie 4.0) sowie die Kompetenzentwicklung, etwa im Rahmen der Programmatik zur "Zukunft der Arbeit“. Entscheidend war darüber hinaus die Unterstützung der internationalen Zusammenarbeit. Kooperationen existieren heute unter anderem mit Frankreich, Tschechien, China und den USA.

Industrie 4.0 hat dazu beigetragen, dass Deutschland sich einen starken industriellen Sektor erhalten hat. 2018 betrug der Anteil der Wertschöpfung in der Industrie am deutschen Bruttoinlandsprodukt $28 \%$ und ist damit deutlich höher als in anderen Industriestaaten wie Frankreich (16,9\%), Großbritannien (18,0\%) oder den USA (18,2\%

20 Vgl. M. Ziesemer: Wer Industrie 4.0 verstehen will, kommt an Deutschland nicht vorbei, in: C. Bär, T. Grädler, R. Mayr (Hrsg.): Digitalisierung im Spannungsfeld von Politik, Wirtschaft, Wissenschaft und Recht, Berlin, Heidelberg 2018, S. 575-583.

21 Vgl. Ernst \& Young: PE 4.0 - How can Financial Sponsors shape tomorrow's Value Creation?, 2017, https://www.ey.com/Publication/ vwLUAssets/ey-pe-4-0-how-can-financial-sponsors-shape-tomorrows-value-creation/\$FILE/ey-pe-4-0-how-can-financial-sponsorsshape-tomorrows-value-creation.pdf (13.12.2019).

22 Vgl. Plattform Industrie 4.0, https://www.plattform-i40.de (12.12.2019). 
im Jahr 2017). ${ }^{23}$ Der weit größere Teil der Wertschöpfung entsteht aber in all diesen Ländern im Dienstleistungssektor, der von digital-gestützten Innovationen bereits heute ähnlich stark verändert wird. Dabei verschmelzen Produktion und Dienstleistungen zunehmend zu einer "hybriden Wertschöpfung" ${ }^{24}$. Die Innovationspolitik kann sich daher nicht allein auf die Industrie konzentrieren.

Eine zentrale Herausforderung über alle Sektoren hinweg ist die zunehmende Bedeutung von Daten als zentraler Wertschöpfungsfaktor. Die Fähigkeit, Daten zu sammeln, auszutauschen, zu verknüpfen und auszuwerten, wird damit zur Schlüsselkompetenz. Daten und Dateninfrastrukturen werden zur "essenziellen Einrichtung“ (essential facility). ${ }^{25}$ Dabei werden heute, auch von deutschen Unternehmen, vorrangig die Infrastrukturen außereuropäischer Anbieter genutzt. Skalierungsvorteile und Netzwerkeffekte verhindern das Entstehen einer umfassenden europäischen Alternative. So ist es Aufgabe des Staats, im Sinne der Technologiesouveränität europäische Wettbewerber zu stärken. Dabei geht es zum einen um den Ausgleich von Nachteilen, die durch eine niedrigere Wagniskapitalverfügbarkeit als auch erhöhte Datenschutzanforderungen entstehen können. Zum anderen können erst dadurch die erwartbar positiven Effekte eines Datenaustauschs nach europäischen Standards realisiert werden, die als externe Effekte bei individuellen strategischen Entscheidungen von Unternehmen in der Regel vernachlässigt werden.

Aus diesen Gründen hat die Bundesregierung im Oktober 2019 Pläne für den Aufbau der europäischen Dateninfrastruktur "GAIA-X“" vorgestellt. ${ }^{26}$ Damit soll ein digitales "Ökosystem" mit standardisierten, nach wohl definierten Regeln zugänglichen und interoperablen Schnittstellen geschaffen werden, in dem Daten sicher und vertrauensvoll verfügbar gemacht, zusammengeführt und geteilt werden können. GAIA-X baut auf Initiativen wie dem „International Data Space“ (vormals "Industrial Data Space“) auf, womit bereits seit 2014 die Entwicklung eines sicheren Datenraums und einer dazu notwendigen Referenzar-

23 Vgl. Weltbank: World Development Indicators, 2019, http://datatopics.worldbank.org/world-development-indicators/ (12.12.2019).

24 Vgl. J. Becker, H. Krcmar: Integration von Produktion und Dienstleistung - Hybride Wertschöpfung, in: Wirtschaftsinformatik, 50. Jg. (2008), S. 169-171.

25 Vgl. Bundesministerium für Wirtschaft und Energie: Ein neuer Wettbewerbsrahmen für die Digitalwirtschaft. Bericht der Kommission Wettbewerbsrecht 4.0, September 2019, https://www.wettbewerbsrecht-40. de/KW40/Redaktion/DE/Downloads/bericht-der-kommission-wettbewerbsrecht-4-0.pdf?__blob=publicationFile\&v=3 (17.12.2019).

26 Vgl. Bundesministerium für Wirtschaft und Energie, Bundesministerium für Bildung und Forschung: Das Projekt GAIA-X - Eine vernetzte Dateninfrastruktur als Wiege eines vitalen, europäischen Ökosystems, 2019, https://www.bmwi.de/Redaktion/DE/Publikationen/Digitale-Welt/dasprojekt-gaia-x.pdf?__blob=publicationFile\&v=22 (12.12.2019). chitektur vorangetrieben wird. ${ }^{27}$ Die Entwicklung solcher Referenzarchitekturen sowie sicherer und standardisierter Technologien, aber auch die Förderung von Anwendungsbeispielen und des Kompetenzaufbaus in den Unternehmen sind wichtige Aufgaben, um das Entstehen eines aktiv genutzten Datenökosystems auf Basis von GAIA-X zu ermöglichen. Sie sind zentrale Elemente einer Forschungs- und Innovationspolitik, die den Umgang mit und die Nutzung von Daten noch stärker in den Fokus nimmt.

Zahlreiche weitere Beispiele verdeutlichen den umfassenden Politikansatz zur Förderung von Technologiesouveränität, orientiert an europäischen Werten und im engen Austausch mit der Gesellschaft. So hat sich die Bundesregierung 2018 mit der nationalen Strategie für Künstliche Intelligenz das Ziel gesetzt, Deutschland zu einem führenden Standort für die Erforschung, Entwicklung und Anwendung von $\mathrm{KI}$ zu machen ${ }^{28}$ und hat innerhalb eines Jahres bereits verschiedene Initiativen umgesetzt: ${ }^{29}$ Technologieentwicklung, z. B. in Kl-Kompetenzzentren, Technologietransfer, z. B. in damit vernetzten Anwendungshubs, sowie die Förderung von $\mathrm{KI}-\mathrm{Kompetenzen} \mathrm{werden} \mathrm{dabei}$ kombiniert mit einem umfassenden Stakeholderprozess im Rahmen der Plattform Lernende Systeme. Und mit dem Rahmenprogramm „Quantentechnologien - Von den Grundlagen zum Markt" treibt die Bundesregierung schon heute intensiv die Forschung und Kompetenzentwicklung in einem Technologiefeld voran, das das Potenzial hat, die Möglichkeiten zur Datenerhebung, Datenübertragung und Datenanalyse auf ein ganz neues Niveau zu heben.

Der Zusammenarbeit über Ressortgrenzen hinweg kommt dabei, gerade in Bezug auf den Technologietransfer, eine Schlüsselrolle zu. Sie ist ein wesentliches Element der deutschen Forschungs- und Innovationspolitik. So haben die Bundesministerien für Bildung und Forschung (BMBF) und für Wirtschaft und Energie (BMWi) seit 2018 gemeinsam den Aufbau einer Agentur zur Förderung von Sprunginnovationen vorangetrieben. Damit soll die Entwicklung von bahnbrechenden technologischen Neuerungen und Geschäftsmodellen und deren Transfer in den Markt gezielt gefördert werden. Die Agentur wurde unter dem $\mathrm{Na}-$ men „SprinD“ im Herbst 2019 gegründet und wird 2020 inre Arbeit aufnehmen. Auch beim Transfer von KI-Methoden in die Nutzung in verschiedenen Branchen arbeiten

27 Vgl. B. Otto et al.: Industrial Data Space. Digitale Souveränität über Daten, Fraunhofer White Paper 2016, https://www.fraunhofer.de/content/dam/zv/de/Forschungsfelder/industrial-data-space/IndustrialData-Space_whitepaper.pdf (12.12.2019).

$28 \mathrm{Vgl}$. Bundesregierung: Strategie Künstliche Intelligenz der Bundesregierung, 2018, https://www.ki-strategie-deutschland.de/home. html?file=files/downloads/Nationale_KI-Strategie.pdf (12.12.2019).

29 Vgl. Bundesregierung: Ein Jahr KI-Strategie, Zwischenbericht, 2019, https://www.ki-strategie-deutschland.de/home.html?file=files/downloads/Zwischenbericht_KI-Strategie_Final.pdf (30.12.2019). 
alle Ressorts gezielt zusammen. ${ }^{30}$ So haben das BMBF, das BMWi sowie das Bundesministerium für Verkehr und Infrastruktur (BMVI) im Juni 2019 gemeinsam den Aktionsplan „Forschung für autonomes Fahren" ${ }^{31}$ auf den Weg gebracht. Dieser bündelt Schwerpunkte und Leitlinien für die künftige Ausrichtung der Forschungsförderung für das autonome Fahren und zeigt Chancen für eine sichere, emissionsarme, intelligente und innovative Mobilität auf. Ein weiteres Beispiel ist der Einsatz digitaler Lösungen im Gesundheitsbereich, der gemeinsam vom BMBF und dem Bundesministerium für Gesundheit (BMG) vorangetrieben wird.

Technologiesouveränität erfordert europäische Zusammenarbeit

Deutschland hat eine gute Ausgangsposition, um das digitale Zeitalter mitzugestalten. Aufgrund seiner relativen Größe wird es aber allein keine entscheidenden Impulse geben können. Denn Standards werden heute maßgeblich durch die Marktmacht von Produzenten und Konsumenten bestimmt. Folglich werden die drei großen Wirtschafts- und Innovationsräume - die USA, Europa und Asien - die Märkte der Zukunft bestimmen.

Dabei hat Europa bereits gezeigt, dass es durch seine Marktmacht Maßstäbe setzen kann, die auch international Wirkung entfalten. Die 2016 in Kraft getretene und ab 2018 in allen Mitgliedstaaten anzuwendende DatenschutzGrundverordnung (DSGVO) gilt heute weltweit als Vorbild, etwa in Kalifornien. ${ }^{32}$ Gleichzeitig haben führende Unternehmen wie Facebook mit Nachbesserungen beim Datenschutz auf die Verordnung reagiert. Auch der Mobilfunkstandard GSM hat gezeigt, wie aus einer europäischen Forschungskooperation durch das Etablieren gemeinsamer, EU-weiter Standards schließlich ein weltweiter Standard durchgesetzt werden konnte.

30 Ebenda.

$31 \mathrm{Vgl}$. Bundesregierung: Aktionsplan Forschung für autonomes Fahren. Ein übergreifender Forschungsrahmen von BMBF, BMWi und BMVI, 2019, https://www.bmbf.de/upload_filestore/pub/Aktionsplan_Forschung_fuer_autonomes_Fahren.pdf (30.12.2019).

32 Vgl. L. Javanshir: The GDPR: It Came, We Saw, but Did It Conquer?, in: Seattle University Law Review, 42. Jg. (2019), H. 3, S. 1019-1021.
Die Förderung der Zusammenarbeit mit europäischen Partnern ist daher ein wichtiger Bestandteil der deutschen Innovationspolitik im digitalen Zeitalter. Dies reicht von multilateralen Kooperationen im Bereich Mikroelektronik, etwa durch das gemeinsame Unternehmen ECSEL (Electronic Components and Systems for European Leadership) und das Ende 2018 gestartete wichtige Vorhaben von gemeinsamem europäischen Interesse (Important Project of Common European Interest, IPCEI), über die Zusammenarbeit mit Frankreich im Bereich KI bis hin zu den neu gestarteten europäischen Maßnahmen im Bereich Quantentechnologien (z. B. QuantERA).

\section{Eine Gestaltung des digitalen Zeitalters ist möglich}

Die Forschungs- und Innovationspolitik verfolgt bereits seit vielen Jahren den Anspruch, die Digitalisierung im Sinne unserer Werte und zum Wohle der Menschen zu gestalten. Dazu setzt sie einen maßgeschneiderten, umfassenden und in Europa verankerten Politikansatz um. Die Vergangenheit hat gezeigt, dass ein solcher Ansatz geeignet ist, dem formulierten Gestaltungsanspruch gerecht zu werden. Dabei stellt uns die Digitalisierung in ihren Wechselwirkungen mit allen Lebensbereichen (Umwelt, Mensch, Gesellschaft und Wirtschaft) kontinuierlich vor neue Herausforderungen. Gerade die immer dringlicher werdende Transformation zur Nachhaltigkeit wirft neue Fragen auf, denen wir uns stellen müssen. ${ }^{33}$ Es gilt daher, den etablierten Politikansatz zielgerichtet weiterzuentwickeln. Das BMBF stellt sich dieser Aufgabe, etwa mit dem Ende 2019 vorgestellten Aktionsplan „Natürlich.Digital. Nachhaltig“34.

Technologiesouveränität ist und bleibt ein wesentlicher Baustein. Dabei geht es nicht um Abschottung, sondern um die Schaffung geeigneter Rahmenbedingungen, um selbstbestimmte Entscheidungen der Bürgerinnen und Bürger zu ermöglichen. Das ist Politik im ordoliberalen Sinne.

33 Vgl. z.B. WBGU, a.a. O.

34 Vgl. Bundesministerium für Bildung und Forschung: Natürlich. Digital. Nachhaltig. Ein Aktionsplan des BMBF, 2019, https://www.bmbf.de/ upload_filestore/pub/Natuerlich_Digital_Nachhaltig.pdf (14.1.2020).

Title: Digital Innovations and Technological Sovereignty

Abstract: Digital innovations are changing our economies and societies. Shaping this transformation in line with core societal values and in a continual exchange with society is a key political challenge. The authors argue that technological sovereignty lies at the heart of this challenge. Rather than the autarchy from foreign technologies, this comprises the ability to define and establish core requirements on and properties of technologies, which safeguard the self-determination of citizens in the digital age. The authors show how an integrated innovation policy, which rests upon European collaboration and combines the promotion of technology development, technology transfer and individuals' competencies, can achieve this goal.

JEL Classification: O32, O33, 038 